\title{
The receptor tyrosine kinase inhibitor vandetanib activates Akt and increases side population in a salivary gland tumor cell line (A253)
}

\author{
YUKA FUJISHIRO $^{1,2}$, MORIO TONOGI ${ }^{1,2}$, HIROMI OCHIAI ${ }^{3}$, KENICHI MATSUZAKA ${ }^{1}$, \\ GEN-YUKI YAMANE ${ }^{1,2}$ and TOSHIFUMI AZUMA ${ }^{1,3}$
}

\begin{abstract}
${ }^{1}$ Oral Health Science Center HRC7, Tokyo Dental College, 1-2-2 Masago, Mihama-ku, Chiba City 261-8502; ${ }^{2}$ Department of Oral Medicine, Oral and Maxillofacial Surgery, Tokyo Dental College, 5-11-13 Sugano, Ichikawa-shi, Chiba City 272-8513;

${ }^{3}$ Department of Biochemistry, Tokyo Dental College, 1-2-2 Masago, Mihama-ku, Chiba City 261-8502, Japan
\end{abstract}

Received January 5, 2012; Accepted March 7, 2012

DOI: 10.3892/ijo.2012.1434

\begin{abstract}
We and others have reported that cancer side population (SP) cells have self-renewal and multidrug resistance capabilities. These phenotypes are similar to those of cancer stem cells (CSCs), cancer stem-like cells and tumor-initiating cells (TICs). It has also been reported that upregulation of the epidermal growth factor receptor (EGFR) significantly increases the number of cancer SP cells, conversely, molecular targeting of EGFR tyrosine kinases using specific kinase inhibitors downregulates CSCs. Thus, we used flow cytometric analysis and cell sorting to examine cancer SP cells in the SCA9.cl-15, WR21 and A253 cell lines that originate from a salivary gland tumor (SGT). We successfully isolated cancer SP cells from all of these cell lines. SP cells were detected following treatment of these cell lines with the receptor tyrosine kinase inhibitors (RTKIs) lapatinib, erlotinib and vandetanib. Several studies reported that RTKIs mostly reduced the SP population in cancer cells. We did not observe any detectable morphological differences between SP cells and non-SP cells. We found that the EGF RTKI lapatinib decreased the number of cancer SP cells in all cell lines investigated; however, the EGF RTKI erlotinib did not cause significant differences in the frequency of cancer SP cells in these cell lines. Addition of vandetanib significantly increased the number of cancer SP cells and upregulated the phosphorylated Akt. As far as we know, this is the first report to show that one of the RTKIs, vandetanib, can activate Akt and increase the number of cancer SP cells. It has been reported that RTKIs could competitively
\end{abstract}

Correspondence to: Dr Toshifumi Azuma, Department of Biochemistry, Tokyo Dental College, 1-2-2 Masago, Mihama-ku, Chiba 261-8502, Japan

E-mail: tazuma@tdc.ac.jp

Key words: cancer stem cells, side population cell, tyrosine kinase inhibitor, epidermal growth factor receptor, vascular endothelial growth factor receptor, PI3K inhibit $\mathrm{ABC}$ transporters and subsequently reduced the number of SP cells. However, our observation indicated that signaling changes induced by RTKIs could even activate Akt and induce the SP population. Investigation of the SP phenotype of SGTs is important for the establishment of optimal cancer therapy.

\section{Introduction}

Many recent studies have suggested that tumor initiating cells (TICs), as well as cancer stem cells (CSCs) or cancer stem-like cells, can be isolated from tumor tissues (1-5). A few TICs are considered to have CSC-characteristics. These cells reside long-term in tissues and would have to self-renew and clonally expand in order to initiate and drive tumor growth. The presence of CSCs in tissues is supported by many reports and many cancers contain a small but significant proportion $(\sim 1 \%)$ of CSCs (6). Moreover, there is increasing evidence to suggest that metastases develop when distant organs are seeded with CSCs. This result implicates CSCs in the initiation and growth of metastatic lesions. CSC-mediated metastases may account for the failure of current anti-tumor treatments to eradicate advanced tumors. Thus, current systematic cancer therapies fail due to their inability to effectively eliminate CSCs (7). There are two reasons for the resistance of CSCs to current anti-tumor therapies. First, CSCs proliferate only infrequently, which would make them relatively resistant to anti-proliferative agents because most current anti-cancer agents rapidly kill proliferating cells. Second, CSCs express multidrug resistant and anti-apoptotic proteins, which further contribute to their resistance to anti-cancer agents. Therefore current chemotherapeutic approaches are not sufficient for elimination of CSCs and an ideal anti-cancer agent should selectively target CSCs. Molecular targeted therapy is a recent development in cancer therapy, and this approach shows great promise for the design of anti-tumor treatments. Receptor tyrosine kinases are attractive targets for drug development since they have been extensively studied and have well described oncogenic properties (8-12). There are currently two major effective anti-tyrosine kinase receptor treatment strategies. One strategy is antibody therapy that targets the extracellular domain of the receptor and another 
strategy is the use of small molecules that compete with ATP for tyrosine kinase activation $(13,14)$. There are extensive clinical trial data regarding the use of small-molecule inhibitors of epidermal growth factor receptor (EGFR) and vascular endothelial growth factor receptor (VEGFR) for many cancers, which have shown several advantages in the use of these molecules for chemotherapy (15-18).

We previously reported that a cancer cell line derived from a head and neck cancer contained side population (SP) cells (19). These cancer SP cells showed self-renewal capacity and generated both SP and non-SP cells. The SP cells derived from cancer cell line also expressed high levels of ABC transporter proteins, which are related to multidrug-resistance (MDR) proteins. The SP cells were quite resistant to anticancer agents, including 5-fluorouracil and carboplatin, and upregulated expression of anti-apoptosis-related genes. We also reported that transforming growth factor- $\beta$ dramatically increases the cancer SP cell population and that this increase is accompanied by upregulation of EGFR expression (20).

Hence, we show that inhibitors of EGFR and VEGFR, molecular targeting agent, possibly regulate cancer SP phenotype, one of the candidates for CSCs or TICs, by regulating signal transductions initiated by EGF or VEGF. Malignant salivary gland tumors (SGT) are relatively rare cancers, accounting for less than $1 \%$ of all cancers and 6 to $7 \%$ of head and neck tumors. SGTs display a heterogeneous histology, are aggressive, and show varied responsiveness to current therapies.

We considered that CSCs should be targeted for anti-tumor therapy of SGTs, since the presence of CSCs in different kind of cancers, regardless of their origin, is believed to be critical for tumor development. Since receptor tyrosine kinase inhibitors are potential candidate tools for effective elimination of CSCs, this study focused on the effects of receptor tyrosine kinase inhibitors on CSCs of SGT.

\section{Materials and methods}

Tyrosine kinase inhibitors (TKI). Vandetanib (Zactima, ZD6474), erlotinib (Tarceva, OSI-774) and Lapatinib (Tykerb, GW572016) were purchased from Toronto Research Chemicals Inc. (Toronto, Canada). Each TKI was dissolved in dimethyl sulfoxide (DMSO) at a stock concentration of $10 \mathrm{mM}$.

Cell lines and cell culture. The A253 (human salivary gland epidermoid carcinoma), WR21 (salivary gland tumor type in was-rap transgenic mice) and SCA9.cl-15 (SCA, mouse submandibular gland undifferentiated carcinoma) cell lines were purchased from the American Type Culture Collection (ATCC) and were cultured on $100 \mathrm{~mm}$ dishes in DMEM (Sigma, St. Louis, MO, USA) supplemented with $10 \%$ fetal bovine serum (FBS) (Invitrogen, Carlsbad, CA, USA), 1\% penicillin and streptomycin (Invitrogen) and $10 \mathrm{mM}$ (4-(2-hydroxyethyl)1-piperazine ethanesulfonic acid (HEPES buffer) (Invitrogen) at $37^{\circ} \mathrm{C}$ in a humidified atmosphere containing $5 \% \mathrm{CO}_{2}$.

Flow cytometry. The cells were removed from the culture dishes with $0.25 \%$ trypsin-EDTA (Invitrogen), were pelleted by centrifugation at 1,200 rpm, washed with phosphate-buffered saline (PBS), and resuspended in $37^{\circ} \mathrm{C}$ Hank's balanced salt solution (HBSS) (Sigma) containing 2\% FBS and $10 \mathrm{mM}$
Table I. FACS analysis of SP cells population.

SP cell population $(\%) \pm \mathrm{SD}$

\begin{tabular}{ll}
\hline $\mathrm{A} 253$ & $0.4 \pm 0.3$ \\
$\mathrm{WR} 21$ & $1.5 \pm 0.4$ \\
$\mathrm{SCA}$ & $4.3 \pm 0.8$ \\
\hline
\end{tabular}

The percentage of SP cells in the total population of each cell line (\% SP). Data are the means \pm SD of at least three independent experiments each performed in triplicate. Cancer SP abundance varied between each cell line examined from $0.4 \pm 0.3 \%$ (A253) to $4.3 \pm 0.8 \%$ (SCA9.cl-15).

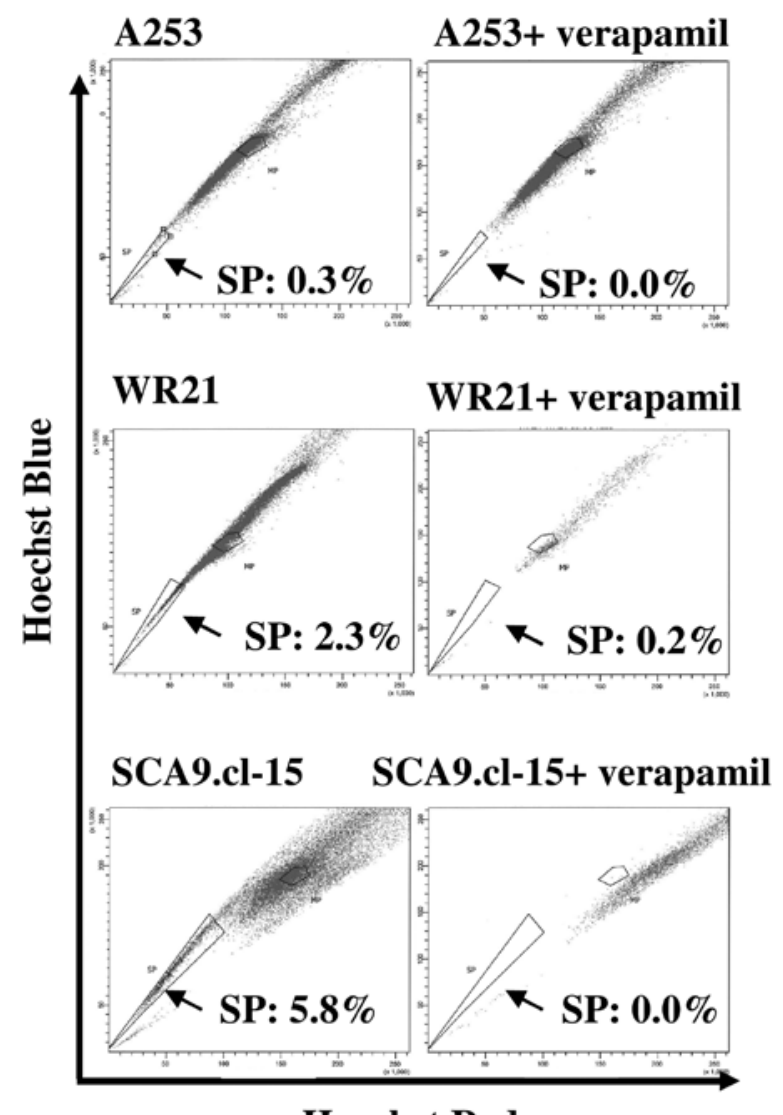

Hoechst Red

Figure 1. Salivary gland tumors contain SP cells. The salivary gland tumor cell lines, SCA9.cl-15 (SCA), A253 and WR21, were stained with Hoechst 33342 and analyzed by dual-wave flow cytometry. SP cells with low blue/red fluorescence were detected in all cell lines. (A) The outlined area indicates the $\mathrm{SP}$ fraction. The percentage of the total cell population that is composed of SP cells is shown. (B) Verapamil treatment significantly reduced the number of SP cells, indicating their dependence on ABC transporter activity. Each sample is representative of 9 samples assayed.

HEPES buffer. The cells $\left(1 \times 10^{6}\right.$ cells $\left./ \mathrm{ml}\right)$ were stained with various concentrations of Hoechst 33342 (Sigma). The stained cells were incubated for $90 \mathrm{~min}$ with or without $50 \mu \mathrm{M}$ verapamil. The cells were then washed with PBS, resuspended in HBSS containing 2\% FBS and $10 \mathrm{mM}$ HEPES, passed through a $40-\mu \mathrm{m}$ mesh filter, and maintained at $4^{\circ} \mathrm{C}$ until analysis. Dead cells were excluded on the basis of propidium iodide staining 
$\mathbf{A}$

A253

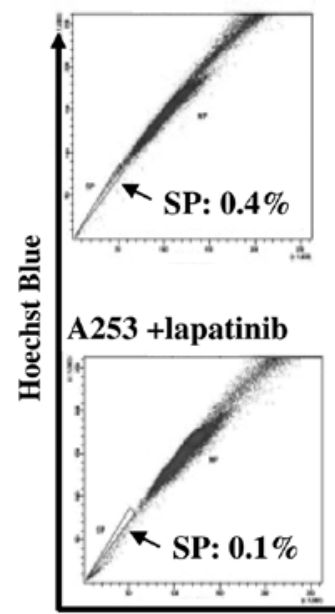

WR21

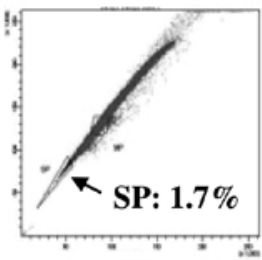

WR21 +lapatinib
SCA9.cl-15

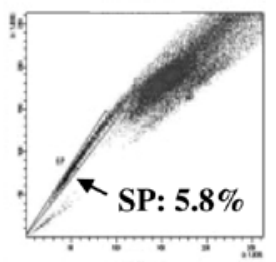

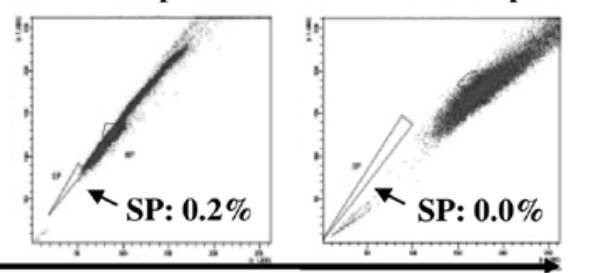

Hoechst Red

\section{B}
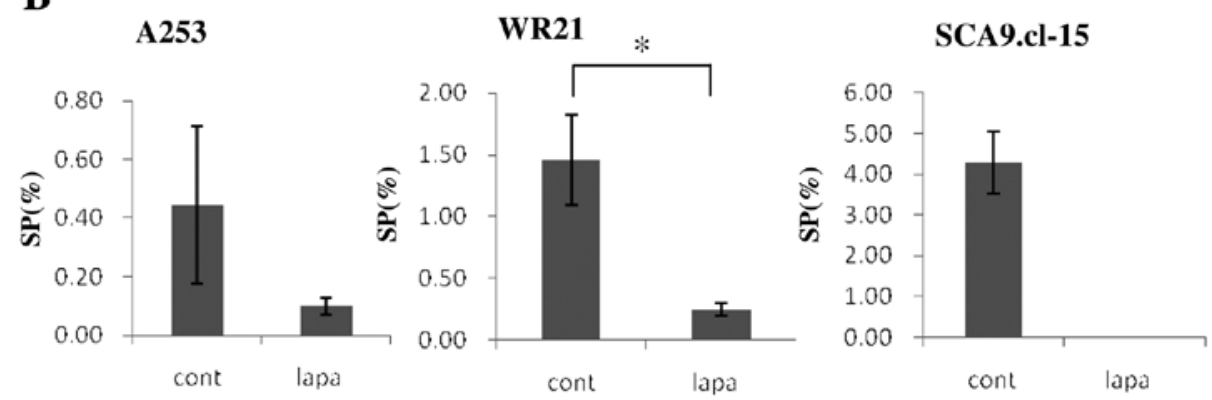

Figure 2. FACS analysis of the effect of lapatinib $(5 \mu \mathrm{M})$ on the SP cell-population. The effect of lapatinib on SP populations of SGT cell lines was determined by FACS analysis of cells treated with or without $5 \mu \mathrm{M}$ lapatinib for $48 \mathrm{~h}$. Lapatinib treatment drastically reduced the number of cancer SP cells in all SGT cell lines. (A) Representative examples of the $5 \mu \mathrm{M}$ lapatinib-induced decrease in the percentage of SP cells within the total population of each cell line. Each sample is representative of 9 samples assayed. (B) Quantification of the SP cell population following treatment of the cell lines with DMSO (cont) or $5 \mu \mathrm{M}$ lapatinib (lapa) for $48 \mathrm{~h}$. At least three independent experiments were performed in triplicate for each cell line. Error bar are means $\pm \mathrm{SD}$. "P<0.05. Lapatanib essentially abolished SP cells in the SCA cell line.

$(2 \mu \mathrm{g} / \mathrm{ml})$ and forward wave scatter. Flow cytometric analysis and cell sorting (FACS) were performed using a dual-laser FACS Aria (Beckton-Dickinson, San Jose, CA, USA). The Hoechst dye was excited at $350 \mathrm{~nm}$ and the fluorescence was measured at two wavelengths, Hoechst blue and red, using 450/50 nm (Hoechst blue) and 530/30 nm (Hoechst red) band pass filters. Hoechst 33342 blue and red fluorescence values are shown on a linear scale and a low value indicates the side population cells.

Tyrosine kinase inhibitor treatment. The cells were seeded at a density of $2.5 \times 10^{6}$ cells in $100-\mathrm{mm}$ dishes in culture medium as described above at $37^{\circ} \mathrm{C}$ in an atmosphere containing $5 \% \mathrm{CO}_{2}$. After $24 \mathrm{~h}$ culture, when the cells were confirmed to be $70 \%$ confluent, the TKIs (erlotinib, lapatinib or vandetanib) were added at a final concentration of $5 \mu \mathrm{M}$ into each culture dish, and the culture was continued as described above for $48 \mathrm{~h}$. The cells were then removed from the dishes and were analyzed by FACS or by western blotting. Cells cultured with DMSO were used as a control.

Protein extraction and immunoblotting. The cells were cultured in $60-\mathrm{mm}$ dishes until $70 \%$ confluent. The culture medium was then changed to medium without serum. After $24 \mathrm{~h}$ culture, each TKI, or control DMSO, was added at a final concentration of $5 \mu \mathrm{M}$ and the cells were cultured for a further $24 \mathrm{~h}$. The cells were then washed once with ice-cold PBS, and immediately lysed with lysis buffer [20 mM Tris $\mathrm{pH}$ 7.6, $150 \mathrm{mM}$ $\mathrm{NaCl}, 1 \% \mathrm{NP}-40,1 \mathrm{mM}$ sodium orthovanadate and complete protease inhibitor mixture (Roche, Basal, Switzerland)]. The protein extracts were collected by centrifugation at $12,000 \mathrm{x} \mathrm{g}$ for $15 \mathrm{~min}$ at $4^{\circ} \mathrm{C}$. Equivalent protein amounts were resolved on NuPAGE 5\% Tris-Acetate gels (Invitrogen) and were then electrophoretically transferred to PVDF membranes. The membranes were blocked with 5\% bovine serum albumin (BSA) in TBST buffer (0.1 M Tris pH 7.6, 0.5 M NaCl, $0.1 \%$ Tween-20) for $1 \mathrm{~h}$ at room temperature. The membranes were probed with the appropriately diluted primary antibody in TBST (anti-Akt rabbit monoclonal antibody, anti-phosphorylated (P) -Akt rabbit monoclonal antibody, anti-ERK1, anti-ERK2 or anti-P-ERK1/2 rabbit monoclonal antibody; all purchased from Cell Signaling Technology Inc., Danvers, MA, USA) followed by alkaline phosphatase (AP)-conjugated secondary antibody diluted in TBST containing 5\% BSA. Bound antibodies were visualized using 5-bromo-4-chloro-3-indolyl phosphate, toluidine salt/ nitro blue tetrazolium chloride BCIP/NBT (Roche). 
$\mathbf{A}$

A253

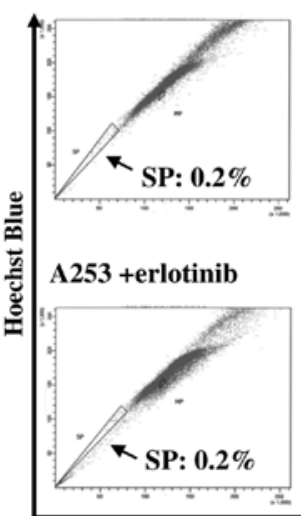

WR21

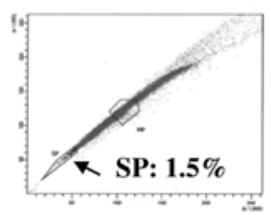

WR21 +erlotinib

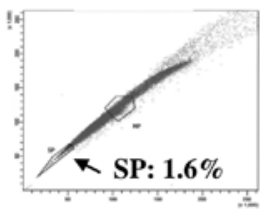

SCA9.cl-15

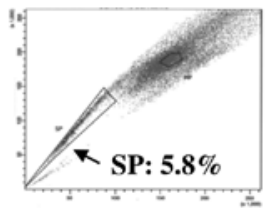

SCA9.cl-15 +erlotinib

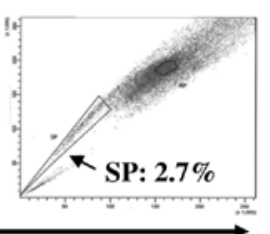

Hoechst Red

B

A253

WR21
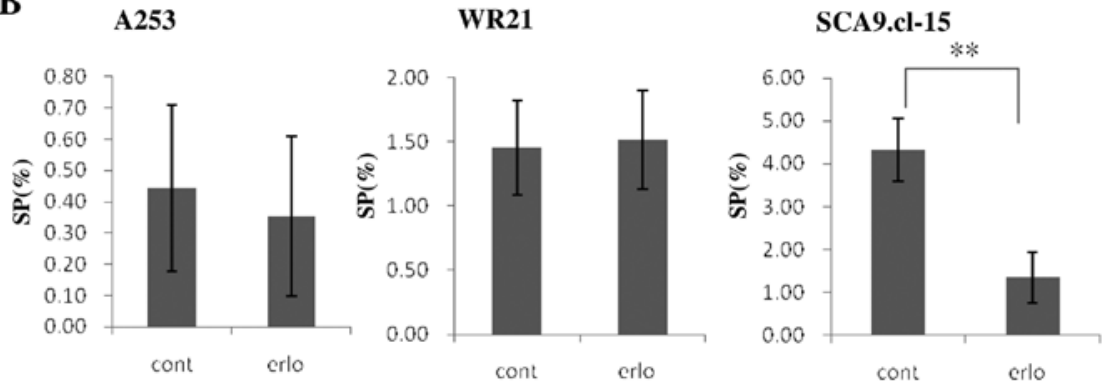

Figure 3.FACS analysis of the effect of erlotinib $(5 \mu \mathrm{M})$ on the SP cell-population. The effect of erlotinib on SP cells was assayed as for lapatinib. (A) Representative examples of the change in the percentage of SP cells within the total population of each cell line following treatment with $5 \mu \mathrm{M}$ erlotinib. Each sample is representative of 9 samples assayed. Erlotinib only dramatically reduced the \% of SP cells in the SCA cell line. (B) Quantification of the SP cell population following treatment of the cell lines with DMSO (cont) or $5 \mu \mathrm{M}$ erlotinib (erlo) for $48 \mathrm{~h}$. At least three independent experiments were performed in triplicate for each cell line. Error bars are means \pm SD. ${ }^{* *} \mathrm{P}<0.01$.

Statistical analysis. Results are reported as means \pm SD. Student's t-test was used to analyze statistical differences between groups. Statistical significance was defined as $\mathrm{p}<0.05$.

\section{Results}

Isolation of SP cells. We used the Hoechst 33342 dye exclusion technique to identify SP cells in SGT cell lines. FACS analysis clearly defined a cancer SP that constituted $0.4 \pm 0.2 \%$ of A253 cells, $1.5 \pm 0.4 \%$ of WR21 cells and $4.3 \pm 0.8 \%$ of SCA cells (Table I). We verified the SP identity of these cells by treatment of the cells with the calcium-channel blocker verapamil hydrochloride followed by FACS analysis (Fig. 1). Verapamil hydrochloride blocks the membrane pump responsible for the SP phenotype. Each experiment was performed at least in triplicate unless otherwise specified. These observations suggested that the cell lines studied do contain SP cells. We could detect no obvious morphological difference between SP and non-SP cells. We also could not identify any cell morphological changes in freshly sorted SP and non-SP cells after seeding and culture for 24 or $48 \mathrm{~h}$, or for 8 days.

Effect of the EGF receptor tyrosine kinase inhibitors, lapatinib and erlotinib on cancer SP cells. Since several reports have shown that EGFR inhibition remarkably reduces the size of cancer SP cell populations, we evaluated the effect of two different selective EGF-receptor tyrosine kinase inhibitors,
A
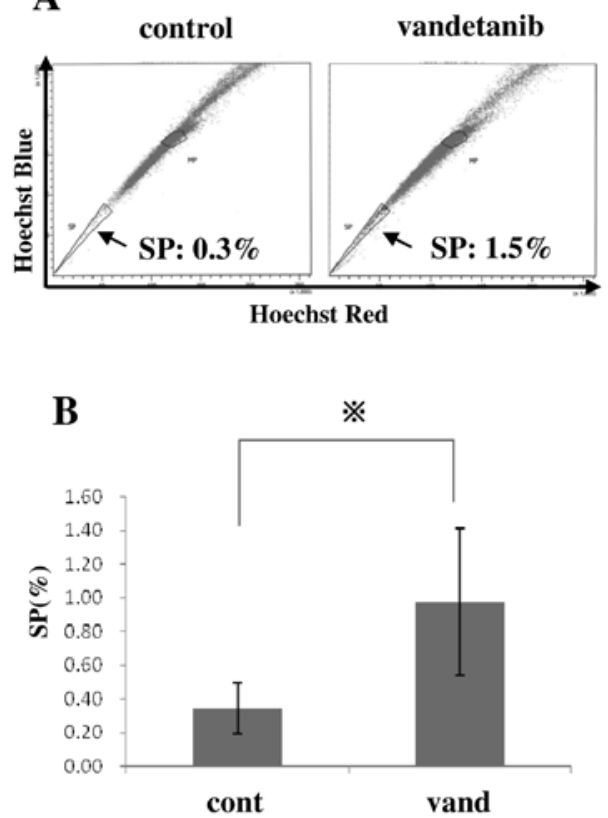

Figure 4. Vandetanib $(5 \mu \mathrm{M})$ increases the percentage of SP cells in the A253 cell line. The effect of vandetanib on SP cells was assayed as for lapatinib. (A) Representative examples of the change in the percentage of SP cells within the total population of the A253 cell line following treatment with $5 \mu \mathrm{M}$ vandetanib. Each sample is representative of 9 samples assayed. (B) Quantification of the SP cell population following treatment of A253 cells with DMSO (cont) or $5 \mu \mathrm{M}$ vandetanib (vand) for $48 \mathrm{~h}$. Three independent experiments were performed in triplicate. Error bar are means $\pm \mathrm{SD} .{ }^{*} \mathrm{P}<0.05$. 


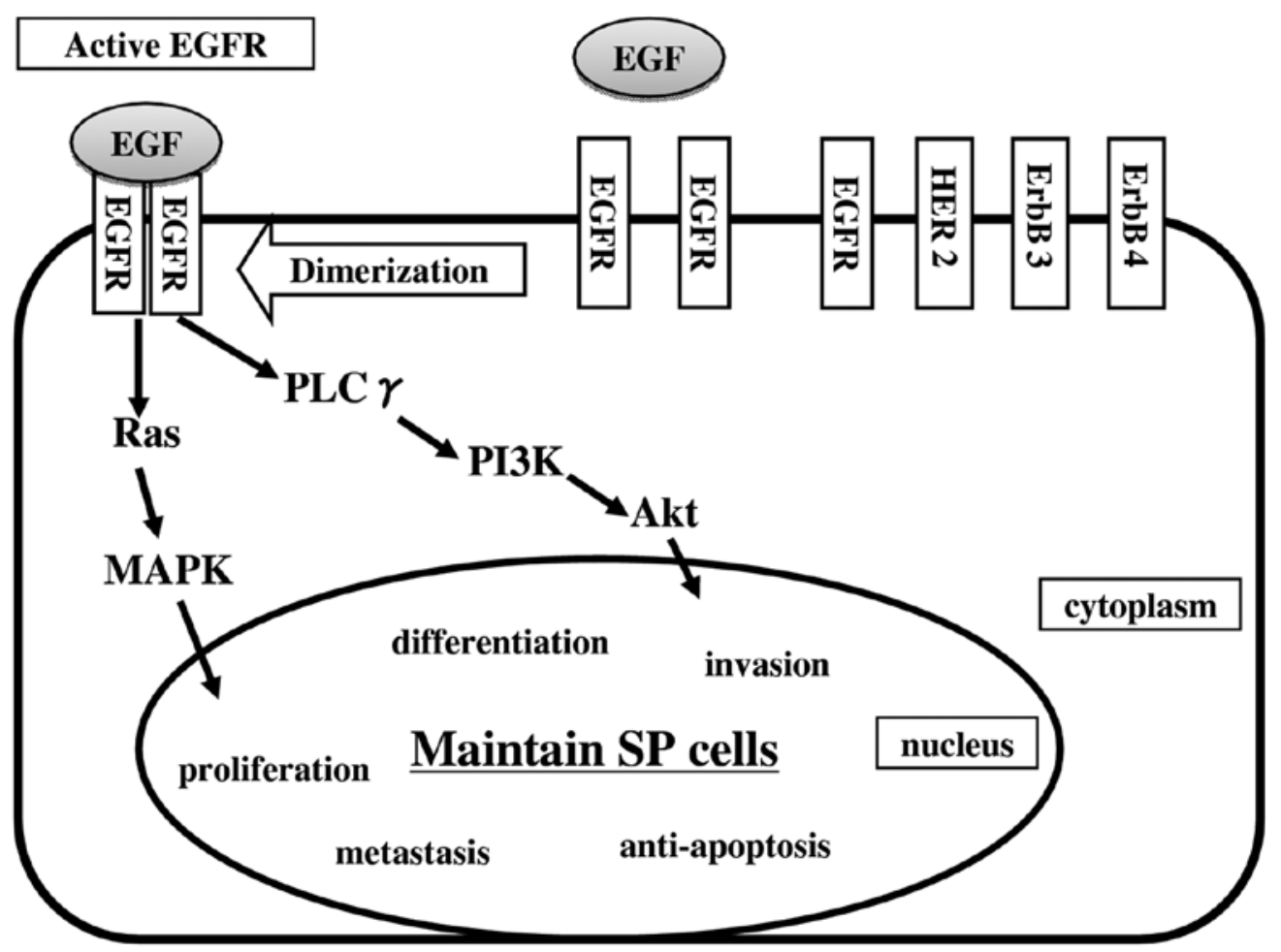

Figure 5. Schematic representation of EGF-receptor family signaling involved in SP cell maintenance. The two key EGFR-family induced signaling pathways believed to be involved in SP cell maintenance are illustrated. These pathways are the MAPK cascade and the PI3K pathway. These pathways are induced by all of the EGF receptor family members shown; EGFR, HER2, ErbB3 and ErbB4. Our experiments using A253 cells indicate that the PI3K pathway plays an important role in maintenance of the SP population.

lapatinib and erlotinib, on SGT cancer cell lines using side population analysis. Lapatinib $(5 \mu \mathrm{M})$ treatment drastically reduced the number of cancer SP cells in all cell lines. Thus, the number of cancer SP cells was significantly lower in A253 cells treated with $5 \mu \mathrm{M}$ lapatinib than in the DMSO-treated control $(0.4 \pm 0.3 \%$ versus $0.1 \pm 0.0 \%)$. SCA cells treated with DMSO had $5.8 \pm 0.8 \%$ cancer SP cells whereas SCA cells treated with $5 \mu \mathrm{M}$ lapatinib had essentially no cancer SP cells. WR21 cells treated with DMSO had $1.5 \pm 0.4 \%$ cancer SP cells, while after treatment with $5 \mu \mathrm{M}$ lapatinib, only $0.2 \pm 0.0 \%$ cancer SP cells were detected (Fig. 2). Treatment with $5 \mu \mathrm{M}$ erlotinib induced little, if any, reduction of SP cells in either A253 or WR21, but did decrease the number of SP cells in the SCA cell line compared to DMSO-treated control cells (Fig. 3).

Effect of the EGF receptor tyrosine kinase inhibitor, vandetanib on cancer SP cells. Vandetanib treatment had no significant effect on the level of SP cells in WR21 cells or in SCA cells. However, we found significantly more SP cells in A253 that were treated with $5 \mu \mathrm{M}$ vandetanib than in A253 that were treated with DMSO (Fig. 4 and Table II).

Western blot analysis of $P$-Akt and P-ERK1/2. Signal transduction pathways are initiated when the EGF receptor tyrosine kinase is activated. One of the major EGF receptor-induced signal transduction pathways involves the activation of phospholipase $\mathrm{C} \gamma$ (PLC $\gamma$ ), Src, PI3K and Akt (Fig. 5). Several reports have indicated that the PI3K and Akt pathway regulates the activity of $\mathrm{ABCG} 2$, which is the key $\mathrm{ABC}$ transporter for
Table II. FACS analysis was performed in the control (DMSO) or vandetanib in three cell lines.

\begin{tabular}{lcc}
\hline & \multicolumn{2}{c}{ SP cell population $(\%) \pm$ SD } \\
& Control & Vandetanib \\
\hline A253 & $0.3 \pm 0.2$ & $1.0 \pm 0.4$ \\
WR21 & $3.7 \pm 1.5$ & $3.7 \pm 2.2$ \\
SCA & $1.0 \pm 0.4$ & $1.3 \pm 1.3$ \\
\hline
\end{tabular}

Vandetanib increases the SP cell population in A253 cells.The effect of $5 \mu \mathrm{M}$ Vandetanib or control DMSO on the SP population of the three tumor cell lines was analyzed by FACS analysis. Data are the means \pm SD of at least three independent experiments each performed in triplicate.

Hoechst 33342 and the SP phenotype. To investigate PI3K activation in TKI-treated cells, we performed western blot analysis of P-Akt. We found that vandetanib induced significant activation of PI3K as judged by a significant increase in P-Akt in A253 cells compared to the DMSO-treated cells. However, treatment with $5 \mu \mathrm{M}$ vandetanib reduced the level of P-Akt in WR21 (Fig. 6). A second major pathway that is modulated by EGFR activation is the MEK-ERK pathway. We also determined whether vandetanib treatment modulated this pathway. We detected a slight reduction in P-ERK1/2 in all cells that were treated with vandetanib compared to DMSO controls, but this decrease was very small in all cases. 
A
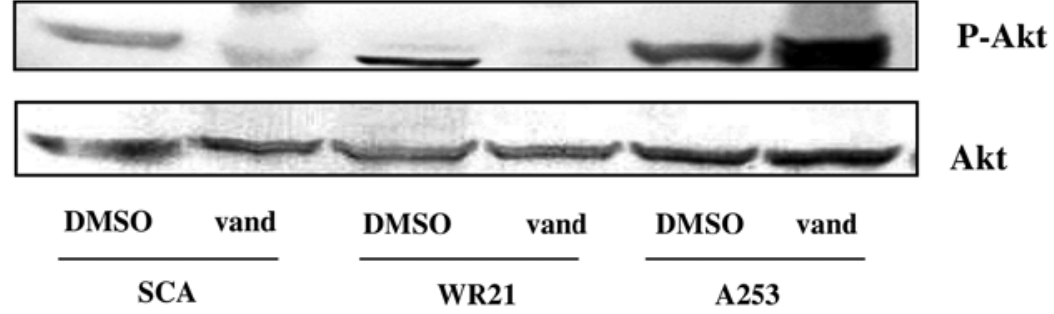

Akt

B

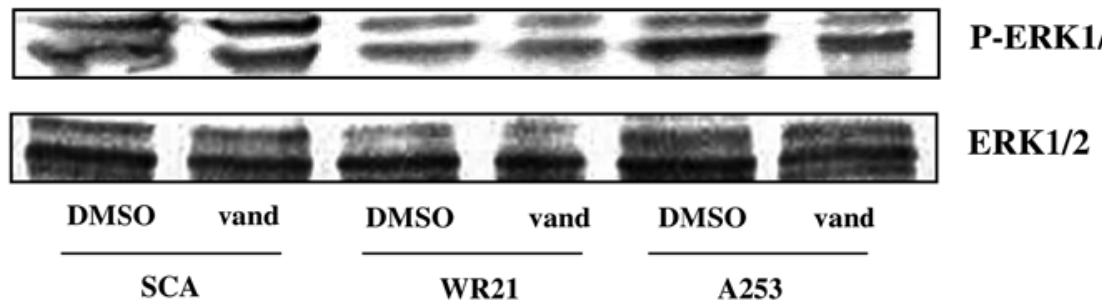

Figure 6. Effect of vandetanib treatment on the phosphorylation of Akt and ERK1/2 in the three tumor cell lines. The effect of $5 \mu \mathrm{M}$ vandetanib (vand), or control DMSO, treatment on the level of phosphorylated (P-)Akt (A) and P-ERK1/2 (B) in the three tumor cell lines was analyzed by western blotting. The total level of Akt and ERK1/2 protein is shown at the bottom. Vandetanib only upregulated P-Akt in A253 cells and had no discernable effect on P-ERK1/2 in any cell line.

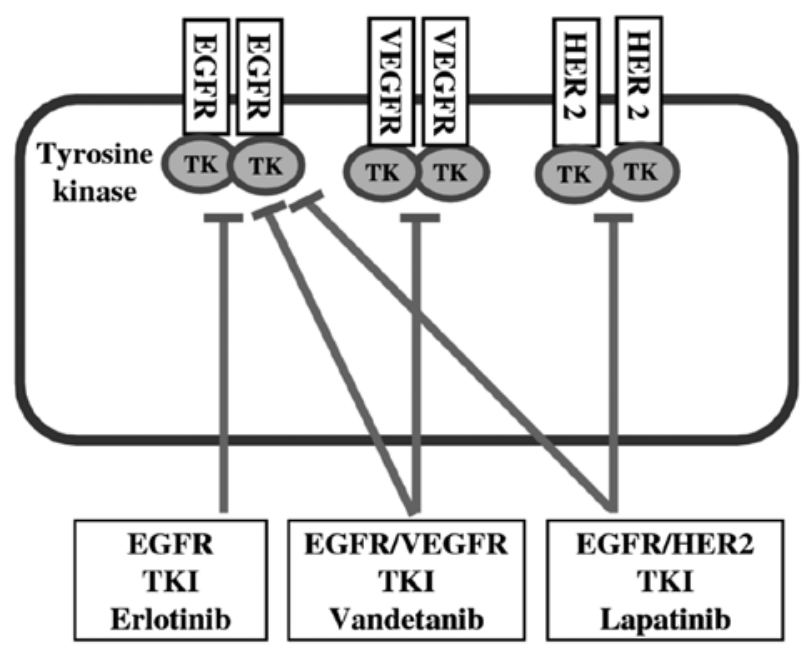

Figure 7. Schematic representation of the dependence of receptor tyrosine kinase inhibition on the affinity of the RTKI for each receptor. Erlotinib, lapatinib and vandetanib have similar molecular structures and they competitively block receptor tyrosine kinases. However, despite this fact, their specificity and affinity for receptors are different as outlined in the scheme.

\section{Discussion}

In this study we found that the effect of TKIs on SP cells may depend on the cellular origin of the side population. These data suggest that the use of TKI for targeted therapy of SP cells requires careful selection of target SP cells.

Molecular targeted therapy is designed to inhibit key signaling pathways in various malignant tumors that are involved in tumor growth and metastasis. The TKIs used in this study inhibit one of the two key molecular targets: EGFR and VEGFR tyrosine kinases (21-24). Several reports have shown that EGFR tyro- sine kinase inhibitors (gefitinib, erlotinib and lapatinib) reverse chemotherapy resistance by blocking ABC transporters (25-29). These kinase inhibitors block ATP-binding by competitively binding to its binding sites. Because the molecular mechanisms of ATP-binding sites of different molecules are similar, one of the major reasons why EGFR kinase inhibitors inhibit ATP transporter effects may be due to direct binding of the EGFR kinase inhibitors to the ATP-binding sites of $\mathrm{ABC}$ transporters. However, we found that not all of these EGF receptor tyrosine kinase inhibitors reduced the number of cancer SP cells. This result indicated that some, but not all, EGF receptor tyrosine inhibitors can inhibit $\mathrm{ABC}$ transporters and reduce the cancer SP cell population. Our data support the possibility that TKIs function through a mechanism other than through direct inhibition of $A B C$ transporters. One such potential mechanism is through inhibition of signal transduction initiated by the EGFR or the VEGFR. As shown in Fig. 7, two key signaling pathways are involved. One pathway is the MAPK cascade (30) and the second is the PI3K pathway $(31,32)$. We found that vandetanib treatment induced the upregulation of PI3K activity in A253 cells, as assessed by an increase in P-Akt (Fig. 7). Bleau et al reported that the PI3K/Akt pathway regulates the cancer SP phenotype and $\mathrm{ABC}$ transporter activity (32). This increased $\mathrm{ABC}$ transporter activity caused an increase in the cancer SP cell population that was accompanied by an increase in P-Akt. Our results suggested that vandetanib induced P-Akt upregulation in A253 resulting in cancer SP phenotypes. Erlotinib, lapatinib and vandetanib have similar molecular structures and they all probably competitively block the EGF-receptor tyrosine kinase, despite the fact that their receptor specificity and affinity are different. Erlotinib only binds to the EGFR (ErbB1). Lapatinib binds to both the EGFR and HER2 (ErbB2), another major receptor for EGF. Vandetanib binds to the EGFR (ErbB1) and the VEGFR (22). All these molecular targeted agents were reported to show clinical promise for the elimination of CSCs. 
In particular, Choi et al showed that vandetanib inhibited the growth of adenoid cystic carcinomas derived from the salivary gland (16). However, our results suggested that vandetanib was the least effective of all of the TKIs tested in reducing cancer SP populations, especially in A253 salivary gland epidermoid carcinoma, and vandetanib may even upregulate these SP populations. These results indicated that vandetanib may not always be effective for eradication of CSCs. As far as we can ascertain, this is the first report that shows that RTKIs can upregulate P-Akt and cancer SP phenotypes. We described above that vandetanib binds and inhibits both EGFR and VEGFR, but, it could activate Akt and increase SP population in A253 cells. These results suggest that EGF receptor-mediated signal transduction plays an important role in the regulation of cancer SP phenotypes that have high chemoresistance.

In conclusion, vandetanib actually increased the number of cancer SP cells in A253 derived from salivary gland and this increase was accompanied by upregulation of PI3K. Therefore, vandetanib has the potential to promote chemoresistance in certain cell types. Investigation of the cancer SP phenotype of SGT may therefore be important for determination of the optimum chemotherapy of these tumors.

\section{Acknowledgements}

We thank Ms. A. Yokoyama for her excellent technical assistance. This research was supported by an HRC7 Oral Health Science Center grant from Tokyo Dental College, by the HighTech Research Center Project for Private Universities, and by a matching subsidy from MEXT (Ministry of Education, Culture, Sports, Science and Technology) of Japan, 2007-2010 (No. 19592414).

\section{References}

1. Wang J, Guo LP, Chen LZ, Zeng YX and Lu SH: Identification of cancer stem cell-like side population cells in human nasopharyngeal carcinoma cell line. Cancer Res 67: 3716-3724, 2007.

2. Cammareri P, Lombardo Y, Francipane MG, Bonventre S, Todaro M and Stassi G: Isolation and culture of colon cancer stem cells. Methods Cell Biol 86: 311-324, 2008.

3. Huang D, Gao Q, Guo L, et al: Isolation and identification of cancer stem-like cells in esophageal carcinoma cell lines. Stem Cells Dev 18: 465-473, 2009.

4. Ma S, Chan KW, Hu L, et al: Identification and characterization of tumorigenic liver cancer stem/progenitor cells. Gastroenterology 132: 2542-2556, 2007

5. Shen G, Shen F, Shi Z, et al: Identification of cancer stem-like cells in the C6 glioma cell line and the limitation of current identification methods. In Vitro Cell Dev Biol Anim 44: 280-289, 2008.

6. Massard C, Deutsch E and Soria JC: Tumour stem cell-targeted treatment: elimination or differentiation. Ann Oncol 17: 1620-1624, 2006.

7. Boman BM and Wicha MS: Cancer stem cells: a step toward the cure. J Clin Oncol 26: 2795-2799, 2008.

8. Van Schaeybroeck S, Karaiskou-McCaul A, Kelly D, Longley D, Galligan L, Van Cutsem E and Johnston P: Epidermal growth factor receptor activity determines response of colorectal cancer cells to gefitinib alone and in combination with chemotherapy. Clin Cancer Res 11: 7480-7489, 2005.

9. Ding XW, Wu JH and Jiang CP: ABCG2: A potential marker of stem cells and novel target in stem cell and cancer therapy. Life Sci 86: 631-698, 2010.

10. Braun AH,Stark K, Dirsch O, HilgerRA, Seeber S and Vanhoefer U: The epidermal growth factor receptor tyrosine kinase inhibitor gefitinib sensitizes colon cancer cells to irinotecan. Anticancer Drugs 16: 1099-1108, 2005.
11. Konecny GE, Venkatesan N, Yang G, et al: Activity of lapatinib a novel HER2 and EGFR dual kinase inhibitor in human endometrial cancer cells. Br J Cancer 98: 1076-1084, 2008.

12. Shipitsin M, Campbell LL, Argani P, et al: Molecular definition of breast tumor heterogeneity. Cancer Cell 11: 259-273, 2007.

13. Hartmann JT, Haap M, Kopp HG and Lipp HP: Tyrosine kinase inhibitors - a review on pharmacology, metabolism and side effects. Curr Drug Metab 10: 470-481, 2009.

14. Cristofanilli M: Novel targeted therapies in inflammatory breast cancer. Cancer 116 (S11): 2837-2839, 2010.

15. Chen JS, Pardo FS, Wang-Rodriguez J, et al: EGFR regulates the side population in head and neck squamous cell carcinoma. Laryngoscope 116: 401-406, 2006.

16. Choi S, Sano D, Cheung M, et al: Vandetanib inhibits growth of adenoid cystic carcinoma in an orthotopic nude mouse model. Clin Cancer Res 14: 5081-5089, 2008.

17. Alferez D, Wilkinson RW, Watkins J, et al: Dual inhibition of VEGFR and EGFR signaling reduces the incidence and size of intestinal adenomas in Apc(Min/+) mice. Mol Cancer Ther 7: 590-598, 2008.

18. Arao T, Yanagihara K, Takigahira M, Takeda M, Koizumi F, Shiratori Y and Nishio K: ZD6474 inhibits tumor growth and intraperitoneal dissemination in a highly metastatic orthotopic gastric cancer model. Int J Cancer 118: 483-489, 2006.

19. Yajima T, Ochiai H, Uchiyama T, Takano N, Shibahara $T$ and Azuma T: Resistance to cytotoxic chemotherapy-induced apoptosis in side population cells of human oral squamous cell carcinoma cell line Ho-1-N-1. Int J Oncol 35: 273-280, 2009.

20. Nishimura T, Azuma T, Yokoyama A, Ochiai H, Saito H and Hibi T: New mechanism of transforming growth factor-beta signaling in hepatoma: Dramatic up-regulation of tumor initiating cells and epidermal growth factor receptor expression. Hepatol Res 39: 501-509, 2009.

21. Normanno N, De Luca A, Bianco C, et al: Epidermal growth factor receptor (EGFR) signaling in cancer. Gene 366: 2-16, 2006.

22. Takahashi $H$ and Shibuya M: The vascular endothelial growth factor (VEGF)/VEGF receptor system and its role under physiological and pathological conditions. Clin Sci (Lond) 109: 227-241, 2005.

23. Nelson $\mathrm{MH}$ and Dolder CR: Lapatinib: a novel dual tyrosine kinase inhibitor with activity in solid tumors. Ann Pharmacother 40: 261-269, 2006.

24. Ryan AJ and Wedge SR: ZD6474-a novel inhibitor of VEGFR and EGFR tyrosine kinase activity. Br J Cancer 92 (Suppl 1): S6-S13, 2005.

25. Siegel-Lakhai WS, Beijnen JH and Schellens JH: Current knowledge and future directions of the selective epidermal growth factor receptor inhibitors erlotinib (Tarceva) and gefitinib (Iressa). Oncologist 10: 579-589, 2005.

26. Kitazaki T, Oka M, Nakamura Y, et al: Gefitinib, an EGFR tyrosine kinase inhibitor, directly inhibits the function of P-glycoprotein in multidrug resistant cancer cells. Lung Cancer 49: 337-343, 2005.

27. Yang $\mathrm{CH}$, Huang CJ, Yang CS, Chu YC, Cheng AL, WhangPeng J and Yang PC: Gefitinib reverses chemotherapy resistance in gefitinib-insensitive multidrug resistant cancer cells expressing ATP-binding cassette family protein. Cancer Res 65: 6943-6949, 2005.

28. Dai CL, Tiwari AK, Wu CP, et al: Lapatinib (Tykerb, GW572016) reverses multidrug resistance in cancer cells by inhibiting the activity of ATP-binding cassette subfamily B member 1 and G member 2. Cancer Res 68: 7905-7914, 2008.

29. Mi Y and Lou L: ZD6474 reverses multidrug resistance by directly inhibiting the function of P-glycoprotein. Br J Cancer 97: 934-940, 2007.

30. Tsuchida R, Das B, Yeger H, et al: Cisplatin treatment increases survival and expansion of a highly tumorigenic side-population fraction by upregulating VEGF/Flt1 autocrine signaling. Oncogene 27: 3923-3934, 2008

31. Zhou J, Wulfkuhle J, Zhang H, et al: Activation of the PTEN/ mTOR/STAT3 pathway in breast cancer stem-like cells is required for viability and maintenance. Proc Natl Acad Sci USA 104: 16158-16163, 2007.

32. Bleau AM, Hambardzumyan D, Ozawa T, Fomchenko EI, Huse JT, Brennan CW and Holland EC: PTEN/PI3K/Akt pathway regulates the side population phenotype and ABCG2 activity in glioma tumor stem-like cells. Cell Stem Cell 4: 226-235, 2009. 